\title{
Sporadic lymphangioleiomyomatosis with bloody sputum as an initial symptom: A case report and review of the literature
}

\author{
KAI WU and HUI-YU LU
}

\author{
Department of Respiratory Medicine, Taizhou People's Hospital, Taizhou, Jiangsu 225300, P.R. China
}

Received July 20, 2014; Accepted March 20, 2015

DOI: $10.3892 /$ etm.2015.2397

\begin{abstract}
Lymphangioleiomyomatosis (LAM) is an extremely rare lung disease affecting females of a childbearing age. The condition occurs sporadically or in association with tuberous sclerosis complex. The diagnosis of LAM is often delayed since the clinical symptoms and signs are non-specific. The present study reports the a case of a patient with LAM, in which bloody sputum was presented as the initial symptom. The 43-year-old female had experienced a small amount of bloody sputum over the previous five years. The patient was admitted to the Department of Respiratory Medicine at Taizhou People's Hospital (Taizhou, China) in 2012, reporting symptoms of a cough, shortness of breath and bloody sputum over the previous 10 days. A high-resolution computed tomography scan revealed multiple small circular thin-walled translucent areas in both lung fields. The initial diagnosis of the patient was LAM. A biopsy was performed using a video-assisted thoracoscopy. In conclusion, increased awareness and early diagnosis and treatment were determined to be key factors in ensuring a satisfactory prognosis.
\end{abstract}

\section{Introduction}

Lymphangioleiomyomatosis (LAM) is a rare, genetically determined, progressive disease that occurs frequently in females of a childbearing age. Sporadic LAM affects one in 400,000 adult females, while the condition occurs in $\sim 40 \%$ of women with tuberous sclerosis complex (TSC) (1). LAM is a slowly progressive lung disease that is associated with mutations in TSC genes. In the disease, neoplastic smooth muscle cells infiltrate the pulmonary parenchyma and lymphatic system, resulting in extensive tissue remodeling and architectural distortion of the lung. In addition, LAM is associated with tumors of the chest and abdomen, including

Correspondence to: Mr. Kai Wu, Department of Respiratory Medicine, Taizhou People's Hospital, 210 Yingchun Road, Taizhou, Jiangsu 225300, P.R. China

E-mail:wk750210@163.com.cn

Key words: lymphangioleiomyomatosis, chylous pleural effusions, diagnosis, therapy lymphangiomyomas and angiomyolipomas (2). A diagnosis of LAM can be confirmed with the presence of pulmonary cysts in computed tomography (CT) scans, or the proliferation of abnormal smooth muscle cells, as detected by a lung biopsy (3). Patients with LAM usually develop progressive dyspnoea and recurrent pneumothorax, chylous collections and occasional hemoptysis (4). In the majority of cases, a biopsy is obtained using video-assisted thoracoscopy. However, misdiagnosis is common and may result in inappropriate therapeutic procedures that can further complicate treatment. The present study reports the case of a patient with sporadic LAM who presented with bloody sputum as the initial symptom, which subsequently resulted in a delay in diagnosis. The current study discusses the diagnosis and treatment protocols of the patient with sporadic LAM, and compares the etiology, diagnosis and treatment of the case with previously reported cases of LAM in the literature.

\section{Case report}

A 43-year-old female had presented with a small amount of bloody sputum over the previous five years. The bloody sputum was considered to be the result of inflammation and was improved following anti-infection and hemostasis treatment. A chest X-ray examination performed in 2009 revealed no evident abnormalities. However, a chest computed tomography (CT) scan performed in the Traditional Chinese Medical Hospital of Taizhou (Taizhou, China) in February 2011 revealed a small area of floc density shadow with a blurred edge in the tip section of the right lung, frosting-like changes in the remaining lung fields and a scattered bullous emphysema shadow in both lung fields (Fig. 1).

The patient was admitted to the Department of Respiratory Medicine at Taizhou People's Hospital (Taizhou, China) in January 2012, reporting symptoms of a cough, shortness of breath and bloody sputum over the previous 10 days. A high-resolution computed tomography (HRCT) scan and an enhanced chest CT scan revealed increasing, thickening and blurred lung markings, ground glass-like changes, multiple small circular thin-walled translucent areas in both lung fields and mild mediastinal lymphadenopathy (Fig. 2). A complete blood test and biochemical tests produced normal results. In addition, tests for exfoliated cells and acid-fast bacilli in the sputum were negative. Color ultrasonography of the spleen, pancreas and gallbladder were normal. However, a uterine, 
ovary and fallopian tube color ultrasonography examination revealed the presence of fibroids in the uterus. Pulmonary function tests revealed a mild obstructive ventilatory dysfunction. After hospitalization, the symptoms were shown to improve following the administration of anti-infection (levofloxacin $0.4 \mathrm{qd}$ and aztreonam $2.0 \mathrm{bid}$ ) and hemostasis (aminomethylbenzoic acid $0.5 \mathrm{qd}$ ) treatment in 10 days. The initial diagnosis of the patient was LAM. However, the patient refused to undergo a further biopsy to confirm the diagnosis.

A positron emission tomography-CT examination performed on February 142012 revealed extensive ground glass-like changes in both lung fields, multiple and scattering diffuse cystic lesions of low density, ground glass nodules with slightly higher intake of 18F-fluorodeoxyglucose (18F-FDG) in the right upper lobe, mediastinal lymph node with no increasing intake of 18F-FDG and uterus fibroids with an increased 18F-FDG intake. In addition, a HRCT scan conducted on February 15 2012 revealed ground glass-like changes and multiple small, circular, thin-walled, translucent areas in both lung fields, small patchy in the right upper pulmonary, mediastinal lymph node (Fig. 3). Tests for the hepatitis B surface antibody and hepatitis B core antibody were positive, whereas the other hepatitis markers were negative. The level of anti-rheumatoid factor was $22 \mathrm{IU} / \mathrm{ml}$, while the level of anti-streptolysin $\mathrm{O}$ was $47 \mathrm{IU} / \mathrm{ml}$. Tests for perinuclear and cytoplasmic anti-neutrophil cytoplasmic antibodies were negative. In addition, tests for anti-nuclear antibodies, such as anti-ribonucleoprotein/Sm, anti-Sm, anti-SSA, anti-SSB, anti-SCl-70 and anti-JO-1 antibodies, were all negative. Estradiol, progesterone and human chorionic gonadotropin levels were within the normal range. High-risk types of human papilloma virus DNA were negative. A transbronchial lung biopsy examination failed to confirm the diagnosis in the Department of Respiratory Medicine. Thus, the patient underwent video-assisted thoracoscopic surgery to perform a resection of the right upper lobe and the right lower lobe tissue on February 29 2012. Pathological analysis confirmed the diagnosis of LAM (Figs. 4 and 5). In addition, immunohistochemistry analysis revealed positive staining for human melanoma black (HMB)-45, melanin A, $\alpha$-smooth muscle actin (SMA; Fig. 6), desmin, CD34 and D2-40, and negative expression for the estrogen receptor (ER) and progesterone receptor (PR). An additional chest X-ray revealed changes following the right lung surgery, which included an increased number of fuzzy lung markings, multiple small, circular, thin-walled, translucent areas in both lung fields, a small amount of pneumothorax and pleural effusion in the right lung on March 19 2012. Blood gas analysis revealed a $\mathrm{pH}$ of $7.44, \mathrm{a} \mathrm{PaO}_{2}$ of $67 \mathrm{mmHg}$ and a $\mathrm{PaCO}_{2}$ of $36 \mathrm{mmHg}$. A small amount of blood remained visible in the sputum, and dyspnea on exertion was observed after discharge. Following discharge the patient was recommended to go to a post-disease clinic of LAM in Zhongshan Hospital (Shanghai, China) but since the patient did not attend, anti-infection and hemostasis treatment was performed in the outpatient clinic of Taizhou People's Hospital (Taizhou, China).

The patient was readmitted to Taizhou People's Hospital on December 32012 due to the presence of blood in the sputum and pain in the right back. Physical examinations revealed a bulging right thorax, percussive flatness in the right lower lung, and significantly lower auscultation of the right side. A chest CT scan of the patient revealed bilateral, diffuse, thin

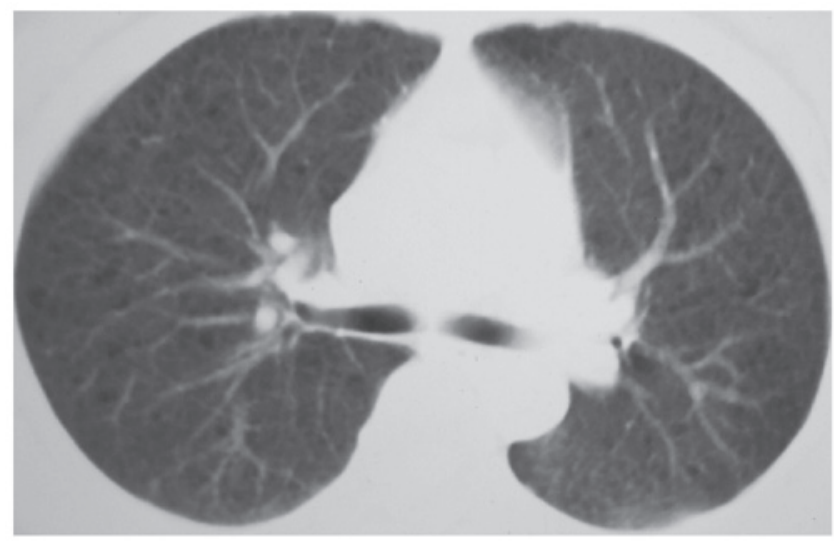

Figure 1. Computed tomography scan showing a scattered, thin-walled balloon shadow in both lung fields.

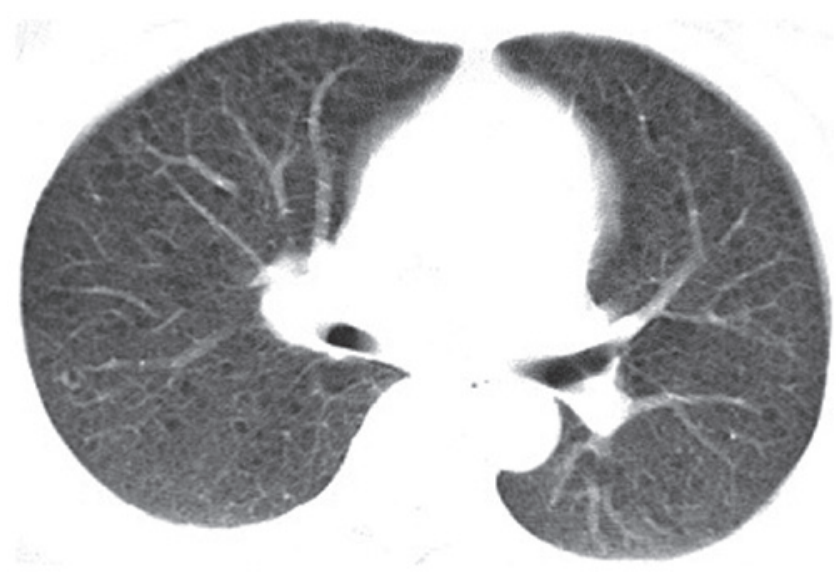

Figure 2. Computed tomography scan showing multiple small, circular, thin-walled, translucent areas in both lung fields.

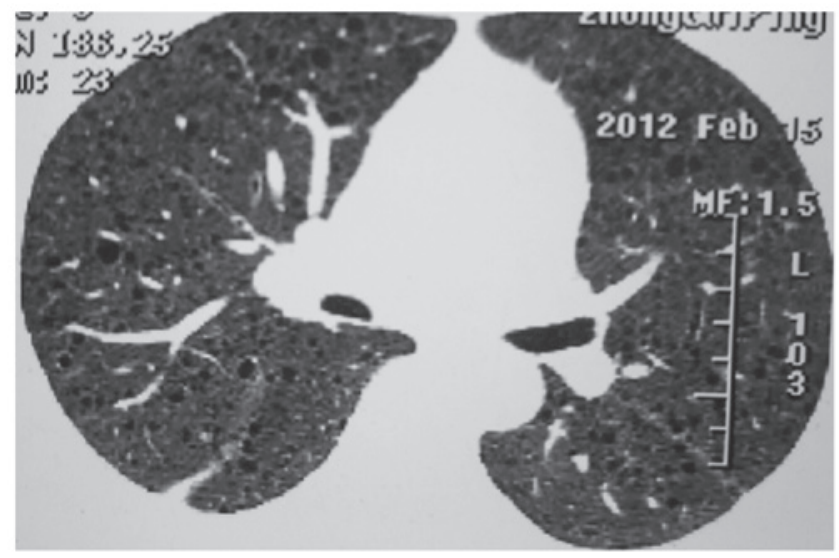

Figure 3. High-resolution computed tomography scan showing ground glass-like changes and multiple small, circular, thin-walled, translucent areas in both lung fields.

cysts within the lung parenchyma, right pleural effusion and right lower lung atelectasis (Figs. 7 and 8). A tube thoracostomy was performed with an ARROW $^{\circledR}$ catheter (Arrow International, Inc., Reading, PA, USA). In total, $\sim 6,000 \mathrm{ml}$ chylous pleural fluid was drained. Rivalta test: positive (+); nucleated cell count, $3.1 \times 10^{9} / 1$; monocyte percentage, $80 \%$; 


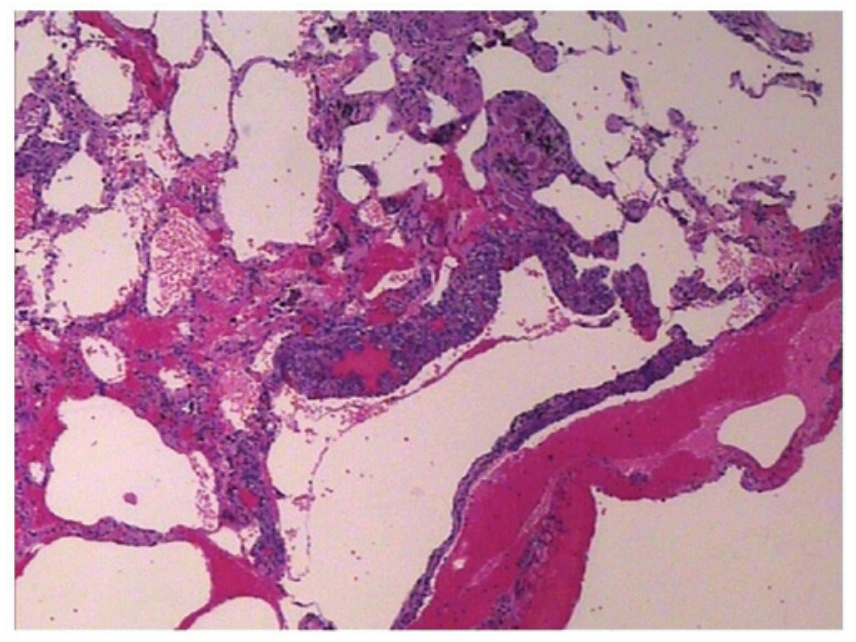

Figure 4. Pathological section showing widened alveolar septum and an enlarged fusion with polycystic changes (hematoxylin and eosin staining; magnification, $\mathrm{x} 40$ ).

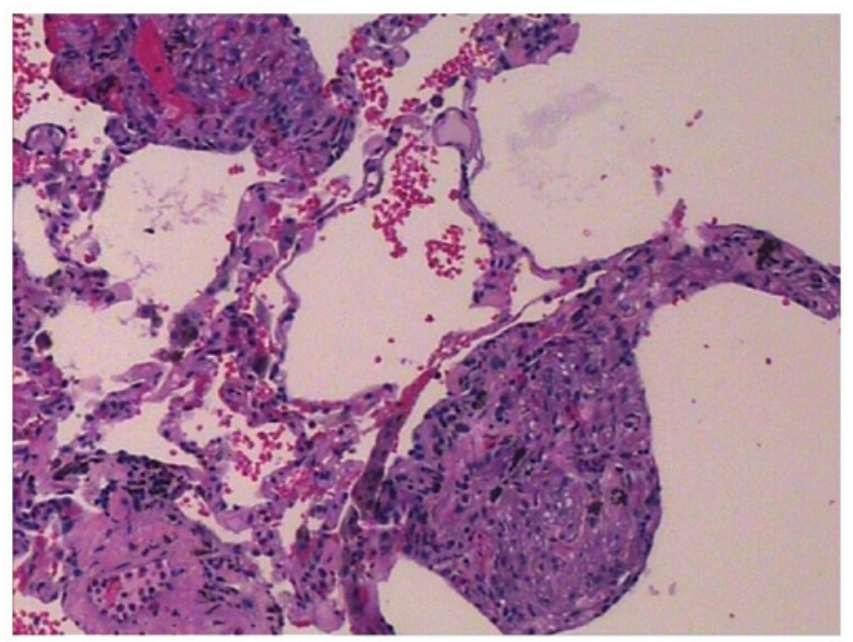

Figure 5. Pathological section showing proliferation and irregular distribution of spindle cells in a widening gap around the thin-walled blood vessels and alveolar wall. Part of the region is nodular form (hematoxylin and eosin staining; magnification, $\mathrm{x} 100$ ).

polynuclear cell percentage, $20 \%$. Biochemistry analysis of the pleural fluid revealed $41.5 \mathrm{~g} / 1$ total protein, $5.4 \mathrm{mmol} / 1$ glucose, $2.55 \mathrm{mmol} / 1$ total cholesterol, $23.6 \mathrm{mmol} / 1$ triglycerides, $101 \mathrm{U} / 1$ lactate dehydrogenase catalase and $5 \mathrm{U} / 1$ adenosine deaminase. Furthermore, blood gas analysis revealed a $\mathrm{pH}$ of 7.39, a $\mathrm{PaO}_{2}$ of $75 \mathrm{mmHg}$ and a $\mathrm{PaCO}_{2}$ of $39 \mathrm{mmHg}$. These examinations were performed on December 72012.

The pleural fluid exudation was significantly reduced following treatment with a fat-free diet, chest tube drainage, anti-infection (mezlocillin sulbactam 5.0 bid) and hemostasis (aminomethylbenzoic acid $0.5 \mathrm{qd}$ ), nutritional supportive treatment in 14 days. Lung function following discharge revealed a mild obstructive ventilatory dysfunction and decreased lung diffusion capacity, as assessed on December 20 2012. A chest X-ray was performed on December 28 2012, which revealed a number of changes following the right lung surgery, including an increased number of fuzzy lung markings, multiple small, circular, thin-walled, translucent areas in both lung fields and

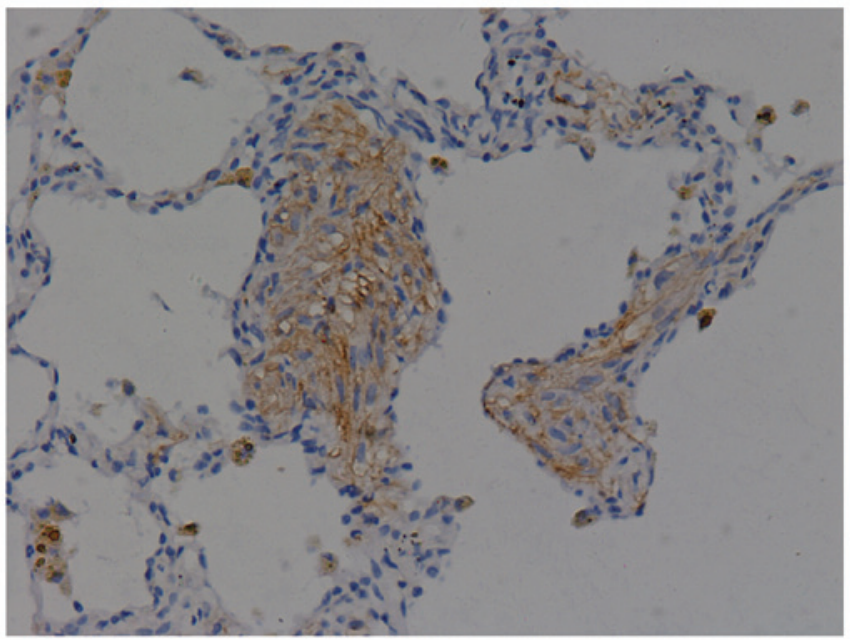

Figure 6. Immunohistochemistry analysis showing positive staining for smooth muscle actin.
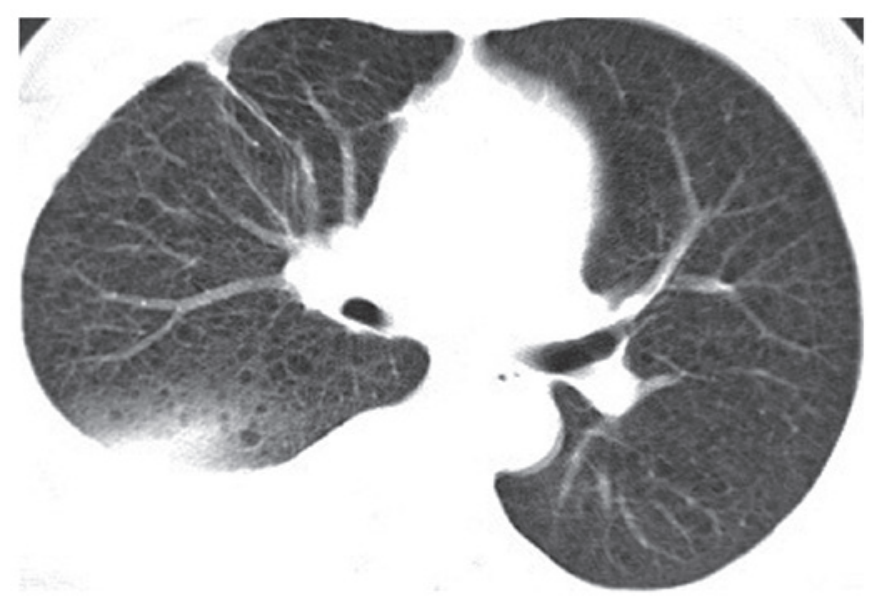

Figure 7. Computed tomography scan showing bilateral, diffuse, thin cysts within the lung parenchyma.

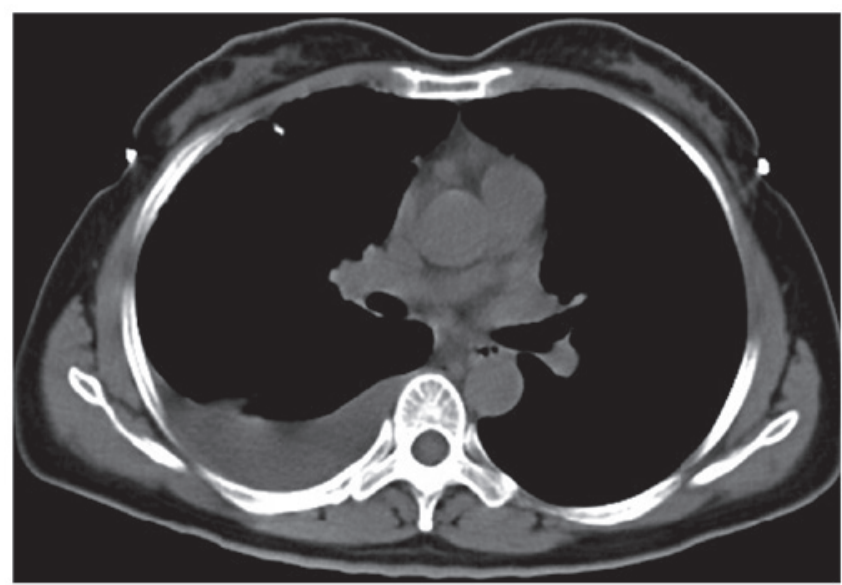

Figure 8. Computed tomography scan showing right pleural effusion and right lower lung atelectasis.

sharp costophrenic angles on both sides of the lungs (Fig. 9). Base on the monitoring of an HRCT scan and pulmonary function test at 3 month intervals, the doctor suggested to use 


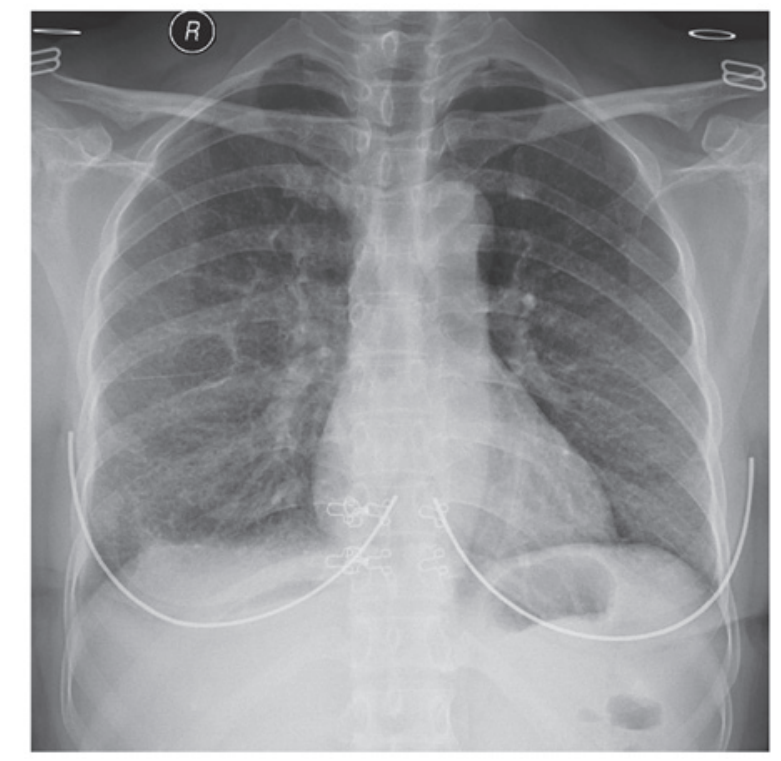

Figure 9. Chest X-ray showing multiple small circular thin-walled translucent areas in both lung fields and sharp costophrenic angles on both sides.

mTOR inhibitors, such as sirolimus, if the patient's condition progressed.

The study was approved by the Ethics Committee of Taizhou People's Hospital (Jiangsu, China) and according to the Declaration of Helsinki. Written informed consent was obtained from the patient.

\section{Discussion}

Lymphangioleiomyomatosis (LAM) is a rare lung disease of unknown etiology. The condition is characterized by cystic remodeling of the lung parenchyma, caused by the proliferation of abnormal smooth muscle-like LAM cells and the presence of extrapulmonary manifestations, including lymphadenopathy, angiomyolipoma and abdominal lymphangioleiomyoma (5). The pulmonary disease is characterized by various symptoms, including dyspnea, pleural effusion, hemoptysis and spontaneous pneumothorax. Chylothorax is one of the most frequent complications in the course of LAM, appearing in up to $30 \%$ of cases. Pulmonary LAM can be diagnosed through characteristic features in high-resolution computed tomography (HRCT) images, or pathological examination via biopsy. The condition can occur sporadically or in association with tuberous sclerosis complex (TSC). Currently, there is no curative treatment for the disease (6). LAM is a rare, slowly progressive lung disease that almost exclusively affects young women of a reproductive age (7).

Progressive dyspnea on exertion, pneumothorax and chylous pleural effusions are the common pulmonary symptoms of LAM, while hemoptysis may be present occasionally (8). Slow and progressive dyspnea is the result of airway obstruction and cystic degradation of the parenchyma (9). Pneumothorax may be associated with the abnormal proliferation of small airway smooth muscle, which leads to the obstruction of distal airways. Spontaneous pneumothorax is the primary symptom of LAM in $\sim 40 \%$ of cases, and $\sim 80 \%$ of patients have experienced pneumothorax in their medical history (10). In addition, chylothorax may be the first symptom of LAM, occurring as a primary manifestation in $\sim 25 \%$ of patients $(11,12)$. The obstruction of the lymphatic system is the most important mechanism underlying the establishment of chylothorax, while the obstruction of blood vessels leads to the formation of focal areas of hemorrhage and hemoptysis (13). The present study reported a sporadic case of LAM, in which the onset symptom was bloody sputum, and the diagnosis of LAM was confirmed 5 years after the initial presentation. A variety of factors, such as the insidious onset, normal performance in the early chest $\mathrm{X}$-ray, no specific symptoms of bloody sputum and clinicians lacking understanding of the disease, resulted in the missed diagnosis and misdiagnosis of the disease.

Observations on a chest X-ray are usually normal, or there may be presentations of pleural effusion and pneumothorax; thus, diagnosis may be difficult in the early stages of the disease. HRCT imaging has the greatest diagnostic value and is necessary to perform for confirmation of diagnosis. In a previous study, the diagnostic accuracy of LAM was found to be $72 \%$ by CT alone (14). The diagnosis of LAM requires a HRCT scan that demonstrates diffuse, round or ovoid, thin-walled cysts, which vary in size between a few millimeters and $3 \mathrm{~cm}$, and are surrounded by normal pulmonary parenchyma, while in advanced stages of the disease, total replacement of the lung tissue may be observed (5). A number of HRCT features characteristic of LAM were outlined in the European Respiratory Society (ERS) criteria, including multiple ( $>10)$, thin-walled, round, well-defined, air-filled cysts with preserved or increased lung volume, with no other significant pulmonary involvement, specifically no interstitial lung disease, with the exception of possible features of multifocal micronodular pneumocyte hyperplasia in patients with TSC. HRCT features are compatible with pulmonary LAM when only a small number of cysts (>2 and $\leq 10$ ), as described, are present (1). The ERS criteria indicate that confirmation of LAM with a lung biopsy is not always necessary when a HRCT scan exhibits the typical LAM image and there is evidence of one of the following: Angiomyolipomas, chylous effusion, probable or definite TSC or lymphangioleiomyomas (1). In the present case, a chest CT scan performed on February 262011 revealed a scattered low-density cystic lesion; however, the patient was misdiagnosed with another disease. The main differential diagnosis is diffuse lung disease that exhibits multiple cysts in the parenchyma, such as idiopathic pulmonary fibrosis, Langerhans cell histiocytosis, emphysema, cystic bronchiectasis and cystic fibrosis of lung. Patients should be suspected of having LAM when they present with typical HRCT changes, pneumothorax or chylothorax in order to avoid misdiagnosis (5). Pulmonary function tests can show an obstructive or restrictive pattern, and the condition is often accompanied with hypoxemia (15). The lung function and blood gas analysis are in line with these changes. Previous studies have shown that the forced expiratory flow in $1 \mathrm{sec}$ (FEV1) and the carbon monoxide lung diffusing capacity (DLCO) often reflect the dynamic changes in disease progression, and are associated with the abnormalities observed in CT and histological examinations in patients with LAM (16-18). Abnormalities in DLCO are more common than in FEV1; thus, DLCO may be a more sensitive indicator of early lesions, while DLCO and FEV1 are likely to be the best current indicators of disease progression and survival (1). 
Pathological examination is very important for the diagnosis of LAM. Two lesions characterize LAM, namely cysts and a multifocal nodular proliferation of immature smooth muscle and perivascular epithelioid cells (LAM cells) (1). LAM cells are smooth muscle cell precursors that stain positive for several smooth muscle markers, including $\alpha$-SMA and desmin, as well as melanocytic markers, such as HMB-45, human melanosome-associated antigen-1 and Melan-A $(5,19)$. LAM cells may also express ER and PR. When the pathological features of LAM are not completely clear, immunohistochemistry of SMA and HMB-45 should be performed to assist in the diagnosis. Immunohistochemistry analysis of SMA, desmin and HMB-45 is an important adjunct to diagnosis $(20,21)$. Since the samples obtained by transbronchial biopsy are generally small, analysis of the HMB-45 marker is particularly important for diagnosis (22). A transbronchial biopsy may be a safe and effective method for establishing the diagnosis of LAM, obviating the requirement for surgical lung biopsy in more than half of LAM patients (23). In the present study, a diagnosis was unable to be confirmed in the patient using a transbronchial lung biopsy; however, the patient subsequently underwent video-assisted thoracoscopic surgery to perform a lung tissue resection. Pathological analysis and immunohistochemical staining combined with the CT examination and clinical manifestations were used to confirm the diagnosis of LAM. In approximately half of LAM cases, the ER and/or PR can be detected by immunohistochemistry (24). Therefore, tests for PR and ER may aid diagnosis, although staining for the ER and PR in the present case was negative.

In the ERS criteria, the gold standard for the diagnosis of LAM is the clinical and histopathological basis of LAM. In the present case, the clinical manifestations of the patient were blood in the sputum, progressive dyspnea on exertion, right chylothorax and hypoxemia. Lung function assessment revealed a mild obstructive dysfunction and decreased diffusion capacity. In such cases, a correlation with clinical features and the CT scan is essential to increase the confidence level of diagnosis. In addition, a lung biopsy fitting the pathological criteria for LAM can further confirm the diagnosis, since the disease can occur sporadically without the presence of TSC. Substantial chylothorax was evident following the video-assisted thoracoscopy in the present case. However, surgery may aggravate the condition, in addition to considering the original pathogenesis.

In the past, LAM was difficult to diagnose and the disease had a mortality rate of $100 \%$ after 10 years; however, more recent statistics show there is a $71 \%$ survival rate after 10 years $(25,26)$. At present, there is no effective treatment for LAM (27). The disease affects females of a reproductive age and deteriorates during pregnancy or therapy with exogenous estrogens and contraceptive pills (28). It is hypothesized that pregnancy in patients with LAM is associated with an increased risk of pneumothorax and chylothorax. These observations have led to several antiestrogenic therapies, including the use of progesterone, gonadotropin-releasing-hormone analogs, such as triptorelin and goserelin, and oophorectomy, which appear to stabilize and improve the disease; however, the effects are imprecise $(5,29-32)$. There are no randomized placebo-controlled trials that have confirmed the efficacy of progesterone and hormonal treatment (33). The molecular basis of LAM has been extensively characterized over the past decade, resulting in the development of a targeted therapy. Recent progress in the understanding of the molecular pathogenesis of LAM and muscle cell biology has provided a foundation for the development of novel therapeutic strategies (27). Inhibitors of mammalian target of rapamycin (mTOR), matrix metalloproteinases and angiogenesis are the most promising areas of research (27). Inherited mutations of the TSC- 1 or TSC- 2 genes cause constitutive activation of the mTOR pathway in LAM. Clinical trials using mTOR inhibitors, such as sirolimus, for the treatment of LAM have been conducted (34), and patients should be encouraged to participate in clinical trials using sirolimus, according to the 2010 ERS criteria. Sirolimus is an immunosuppresant approved by the FDA that functions to inactivate the mTOR complex by imitating tuberin, and as a result inhibits cell proliferation (35). Recent studies have shown a reduction of chylous effusions and improvement of the lung function during sirolimus treatment in lymphangioleiomyomas.

Sirolimus may be considered on an individual basis in patients with a rapid decline in lung function or symptoms, after careful evaluation of the risk/benefit ratio in an experienced center. When sirolimus is used, the effect of therapy should be carefully monitored for tolerance and effect on lung function at 3 monthly intervals. The Multicenter International LAM Efficacy of Sirolimus study demonstrated that therapy with rapamycin for one year induced stabilization in the FEV1 and forced vital capacity, a reduction in the serum levels of vascular endothelial growth factor-D, and an improvement of symptoms and patient quality of life. However, following discontinuation of the treatment, the benefits of rapamycin were reversed after 24 months (36). An open-label, non-randomized, within-subject, dose escalation, safety, tolerability and efficacy study of everolimus (a second generation mTOR inhibitor) in females with sporadic or TSC-LAM is ongoing (27). Patients with severe or rapidly progressing LAM may benefit from a lung transplantation. LAM recurring in the transplanted lung following a single or bilateral lung transplant is rare and generally asymptomatic.

In conclusion, women of a child-bearing age with symptoms of pneumothorax, hemoptysis, progressive dyspnoea and chylous pleural effusions may be suffering from LAM. A HRCT and lung biopsy should be performed in time, and a lung biopsy can be used to decrease the rate of misdiagnosis. In this situation, the increased awareness of diagnosis and knowledge with regard to the clinical presentations of LAM are key factors in ensuring an immediate diagnosis and adequate intervention. All patients diagnosed with LAM should be referred to a LAM treatment center to receive improved guidance with regard to the treatment options available (37).

\section{References}

1. Johnson SR, Cordier JF, Lazor R, et al; Review Panel of the ERS LAM Task Force: European Respiratory Society guidelines for the diagnosis and management of lymphangioleiomyomatosis. Eur Respir J 35: 14-26, 2010.

2. Meraj R, Wikenheiser-Brokamp KA, Young LR and McCormack FX: Lymphangioleiomyomatosis: new concepts in pathogenesis, diagnosis, and treatment. Semin Respir Crit Care Med 33: 486-497, 2012. 
3. Pallisa E, Sanz P, Roman A, et al: Lymphangioleiomyomatosis: pulmonary and abdominal findings with pathologic correlation. Radiographics 22: S185-S198, 2002.

4. Johnson S: Rare diseases. 1. Lymphangioleiomyomatosis: clinical features, management and basic mechanisms. Thorax 54 254-264, 1999.

5. Mavroudi M, Zarogoulidis $\mathrm{P}$, Katsikogiannis $\mathrm{N}$, et al: Lymphangioleiomyomatosis: current and future. J Thorac Dis 5 : 74-79, 2013.

6. Hancock E and Osborne J. Lymphangioleiomyomatosis: a review of the literature. Respir Med 96: 1-6, 2002.

7. Chu SC, Horiba K, Usuki J, et al: Comprehensive evaluation of 35 patients with lymphangioleiomyomatosis. Chest 115 1041-1052, 1999.

8. Johnson SR: Lymphangioleiomyomatosis. Eur Respir J 27: 1056-1065, 2006.

9. Ansótegui Barrera E, Mancheño Franch N, Vera-Sempere F and Padilla Alarcón J: Lymphangioleiomyomatosis. Arch Bronconeumol 47: 85-93, 2011 (In English and Spanish).

10. Almoosa KF, Ryu JH, Mendez J, et al: Management of pneumothorax in lymphangioleiomyomatosis: effects on recurrence and lung transplantation complications. Chest 129: 1274-1281, 2006.

11. Johnson SR and Tattersfield AE: Clinical experience of lymphangioleiomyomatosis in the UK. Thorax 55: 1052-1057, 2000.

12. Ryu JH, Doerr CH, Fisher SD, et al: Chylothorax in lymphangioleiomyomatosis. Chest 123: 623-627, 2003.

13. Juvet SC, McCormack FX, Kwiatkowski DJ, et al: Molecular pathogenesis of lymphangioleiomyomatosis: lessons learned from orphans. Am J Respir Cell Mol Biol 36: 398-408, 2007.

14. Koyama M, Johkoh T, Honda O, et al: Chronic cystic lung disease: diagnostic accuracy of high-resolution CT in 92 patients. AJR Am J Roentgenol 180: 827-835, 2003.

15. Taveira-DaSilva AM, Stylianou MP, Hedin CJ, et al: Decline in lung function in patients with lymphangioleiomyomatosis treated with or without progesterone. Chest 126: 1867-1874, 2004.

16. Johnson SR and Tattersfield AE. Decline in lung function in lymphangioleiomyomatosis: relation to menopause and progesterone treatment. Am J Respir Crit Care Med 160: 628-633, 1999.

17. Avila NA, Chen CC, Chu SC, et al: Pulmonary lymphangioleiomyomatosis: correlation of ventilation-perfusion scintigraphy, chest radiography, and $\mathrm{CT}$ with pulmonary function tests. Radiology 214: 441-446, 2000.

18. Taveira-DaSilva AM, Hedin C, Stylianou MP, et al: Reversible airflow obstruction, proliferation of abnormal smooth muscle cells, and impairment of gas exchange as predictors of outcome in lymphangioleiomyomatosis. Am J Respir Crit Care Med 164: 1072-1076, 2001.

19. Krymskaya VP: Smooth muscle-like cells in pulmonary lymphangioleiomyomatosis. Proc Am Thorac Soc 5: 119-126, 2008.

20. Yu J, Astrinidis A, Howard S, et al: Estradiol and tamoxifen stimulate LAM-associated angiomyolipoma cell growth and activate both genomic and nongenomic signaling pathways. Am J Physiol Lung Cell Mol Physiol 286: L694-L700, 2004.
21. Gao J, Zhu P, Zhang S, et al: A clinicopathological analysis of pulmonary lymphangioleiomyomatosis. Zhongguo Fei Ai Za Zhi 14: 378-382, 2011 (In Chinese).

22. Bonetti F, Chiodera PL, Pea M, et al: Transbronchial biopsy in lymphangiomyomatosis of the lung. HMB45 for diagnosis. Am J Surg Pathol 17: 1092-1102, 1993.

23. Meraj R, Wikenheiser-Brokamp KA, Young LR, et al: Utility of transbronchial biopsy in the diagnosis of lymphangioleiomyomatosis. Front Med 4: 395-405, 2012.

24. Glassberg MK, Elliot SJ, Fritz J, et al: Activation of the estrogen receptor contributes to the progression of pulmonary lymphangioleiomyomatosis via matrix metalloproteinase-induced cell invasiveness. J Clin Endocrinol Metab 93: 1625-1633, 2008.

25. Riojas RA, Bahr BA, Thomas DB, et al: A case report of lymphangioleiomyomatosis presenting as spontaneous pneumothorax. Mil Med 177: 477-480, 2012.

26. Matsui K, Beasley MB, Nelson WK, et al: Prognostic significance of pulmonary lymphangioleiomyomatosis histologic score. Am J Surg Pathol 25: 479-484, 2001.

27. Harari S, Torre O and Moss J: Lymphangioleiomyomatosis: what do we know and what are we looking for? Eur Respir Rev 20: 34-44, 2011.

28. Yano S: Exacerbation of pulmonary lymphangioleiomyomatosis by exogenous oestrogen used for infertility treatment. Thorax 57 : 1085-1086, 2002.

29. Johnson SR and Tattersfield AE. Clinical experience of lymphangioleiomyomatosis in the UK. Thorax 55: 1052-1057, 2000.

30. Fujimoto M, Ohara N, Sasaki H, et al: Pregnancy complicatedwith pulmonary lymphangioleiomyomatosis: case report. Clin Exp Obstet Gynecol 32: 199-200, 2005.

31. Brunelli A, Catalini G and Fianchini A. Pregnancy exacerbating unsuspected mediastinal lymphangioleiomyomatosis and chylothorax. Int J Gynaecol Obstet 52: 289-290, 1996.

32. McLoughlin L, Thomas G and Hasan K. Pregnancy and lymphangioleiomyomatosis: anaesthetic management. Int $\mathrm{J}$ Obstet Anesth 12: 40-44, 2003.

33. Casanova A and Ancochea J: Lymphangioleiomyomatosis: new therapeutic approaches. Arch Bronconeumol 47: 579-580, 2011 (In Spanish).

34. Bissler JJ, McCormack FX, Young LR, et al: Sirolimus for angiomyolipoma in tuberous sclerosis complex or lymphangioleiomyomotosis. N Engl J Med 358: 140-151, 2008.

35. Casanova A, María Girón R, Acosta O, Barrón M, Valenzuela C and Ancochea J: Lymphangioleiomyomatosis treatment with sirolimus. Arch Bronconeumol 47: 470-472, 2011 (In Spanish).

36. McCormack FX, Inoue Y, Moss J, et al; National Institutes of Health Rare Lung Diseases Consortium; MILES Trial Group: Efficacy and safety of sirolimus in lymphangioleiomyomatosis. N Engl J Med 364: 1595-1606, 2011.

37. Kinder B and McCormack FX: Clinical trials for rare lung diseases; lessons from lymphangioleiomyomatosis. Lymphat Res Biol 8: 71-79, 2010. 